\title{
Steady motion of skyrmions and domains walls under diffusive spin torques
}

\author{
Ricardo Gabriel Elías, ${ }^{1, *}$ Nicolas Vidal-Silva, ${ }^{1}$ and Aurélien Manchon ${ }^{2}$ \\ ${ }^{1}$ Departamento de Física and CEDENNA, Universidad de Santiago de Chile, Avda. Ecuador 3493, Santiago, Chile \\ ${ }^{2}$ Physical Science and Engineering Division (PSE), King Abdullah University of Science and Technology (KAUST), Thuwal 23955-6900, \\ Kingdom of Saudi Arabia
}

(Received 15 July 2016; revised manuscript received 7 February 2017; published 8 March 2017)

\begin{abstract}
We explore the role of the spin diffusion of conducting electrons in two-dimensional magnetic textures (domain walls and skyrmions) with spatial variation of the order of the spin precession length $\lambda_{\text {ex }}$. The effect of diffusion reflects in four additional torques that are third order in spatial derivatives of magnetization and bilinear in $\lambda_{\text {ex }}$ and in the nonadiabatic parameter $\beta^{\prime}$. In order to study the dynamics of the solitons when these diffusive torques are present, we derive the Thiele equation in the limit of steady motion and we compare the results with the nondiffusive limit. When considering a homogenous current these torques increase the longitudinal velocity of transverse domain walls of width $\Delta$ by a factor $\left(\lambda_{\text {ex }} / \Delta\right)^{2}(\alpha / 3), \alpha$ being the magnetic damping constant. In the case of single skyrmions with core radius $r_{0}$ these new contributions tend to increase the Magnus effect in an amount proportional to $\left(\lambda_{\mathrm{ex}} / r_{0}\right)^{2}\left(1+2 \alpha \beta^{\prime}\right)$.
\end{abstract}

DOI: 10.1103/PhysRevB.95.104406

\section{INTRODUCTION}

The motion of magnetic textures by electrical currents has attracted a lot of attention in the past ten years, mainly because of a highly rich dynamics and potential applications such as racetrack memory [1] and nonvolatile memory [2]. In the field of spintronics [3], the interaction between magnetic textures hosted in ferromagnetic materials and electric currents in the bulk is a field at the boundary of quantum and semiclassical mechanics [3-5]. The metallic nature of transition metal ferromagnets enables the coupling between the spin angular momentum of transport electrons with the local magnetization. Such a coupling results in the celebrated mechanism of spin transfer torque [6-8]. The main theoretical challenge in the physics of magnetization-current interaction is the comprehensive description of the whole phenomenon within one single model accounting for the micromagnetic dynamics of the texture by the Landau-Lifshitz-Gilbert (LLG) equation [9] as well as the microscopic (quantum or semiclassical) nature of the spin transport [10-12]. Of particular interest is the interaction of the current with magnetic solitons, which have topological stability [13]. Solitons with nontrivial magnetic topology, such as vortices [14] and skyrmions [15-19], have attracted special interest lately due to their potential robustness against pinning and disorder and have been proposed as a promising bit of information for ultradense data storage [20,21].

Several approaches have been proposed [22-25] to describe the effects of currents in magnetic textures. In most of them the $s$ - $d$ model is used. In this model, electrons responsible for the magnetization and electrons responsible for spin transport are artificially separated. This is justified by the fact that the electrons carrying the current are delocalized and occupy mostly the $s$ orbitals while the electrons whose spins produce the magnetization are localized in each molecular site because they mostly occupy $d$ orbitals. The following step is to consider the timescale separation between conduction electrons and molecular spin: While the conduction electrons

\footnotetext{
*gabriel.elias@usach.cl
}

lose quantum coherence within a few femtosecond $\left(\sim \hbar / E_{f}\right.$, where $\hbar$ is the reduced Planck constant and $E_{f}$ the Fermi energy of the conduction electrons), their spin precesses about the local magnetization with a characteristic time about a few tens of femtosecond $\left(\sim \hbar / \Delta_{\mathrm{ex}}\right.$, with $\Delta_{\mathrm{ex}}$ the $s$ - $d$ exchange energy), and lose their spin memory after a few picoseconds, typically. In contrast, the collective magnetization dynamics is not faster than a few hundred picoseconds. As a result, spin transport can be modeled semiclassically, considering a static magnetic texture. When spin-polarized electrons flow in a magnetic texture, at the first order and due to their very fast spin precession, their spin remains aligned on the local magnetization direction. This results in a spin transfer torque, or adiabatic torque, $\sim \nabla \boldsymbol{m}$ [26]. At the second order, when accounting for the actual precession of the spin momentum about the magnetization, another torque emerges, called the nonadiabatic torque $\sim \boldsymbol{m} \times \nabla \boldsymbol{m}$ [23,26-28]. The next order terms enter in the analysis when the variation of the magnetization occurs on scales corresponding to the spin diffusion length $[10,29,30]$. The study of spin diffusion began with the works about spin accumulation [31,32]. This effect is characterized by the diffusion of the spin density coming from the behavior of the electrochemical potential at the interfaces [32]. Later, the effect was observed shortly after the discovery of the giant-magnetoresistence (GMR) effect [33] with the measure of distance over which electronic spin flips [34]. This distance was called spin diffusion length $\lambda_{\text {sf }}$ and its origin was attributed to the diffusive behavior of the spin density (characterized by the diffusion constant $D_{0}$ ) and the flipping of the spin in a characteristic time $\tau_{\mathrm{sf}}$ as $\lambda_{\mathrm{sf}}=\sqrt{D_{0} \tau_{\mathrm{sf}}}$ [35]. As a matter of fact, in disordered metals that are magnetic momentum scattering leads to the diffusion of nonequilibrium spin density on a distance given by the spin diffusion length (sometimes also called spin relaxation length). In this paper we call spin diffusion all the phenomena coming from the variations of the nonequilibrium part of the spin density which appear in the spin density continuity equation as a diffusive term. In sharp domain walls such as magnetic vortices or perpendicularly magnetized materials, the distance over which the magnetization changes (from $1 \mathrm{~nm}$ for Bloch 
walls in $\mathrm{FePt}$ [36] to $10 \mathrm{~nm}$ for vortices in $\mathrm{NiFe}$ [37]) is much shorter than the mean free path, so that the transport is not diffusive in the texture. In contrast, in smooth magnetic textures spin diffusion is important as the typical size of the magnetic texture exceeds the mean free path. For instance, in $\mathrm{NiFe}$ the domain wall width can be as large as about $\sim 100 \mathrm{~nm}$ [38], while the mean free path does not exceed $15 \mathrm{~nm}$; more interestingly, magnetic skyrmions obtained in transition metal multilayers have a radius ranging from $40-200 \mathrm{~nm}[16,17]$. In these two cases, spin diffusion is expected to become important. In the original works on spin transfer torque in magnetic textures [26,27], the influence of spin diffusion was neglected because the torque was derived to the lowest order in magnetization gradient. However, recent works $[10,29,30]$ have shown that in the case where the characteristic length of the texture is of the order of the spin precession length, spin diffusion can significantly enhance the current-driven torque.

In this work we deduce the torques from the general theory of Zhang and $\mathrm{Li}$ [26] and we explore their impact on selected two-dimensional magnetic solitons. An important class of magnetic textures in one and quasi-one dimensions are the so-called domain walls (DWs). These DWs are regions of varying magnetization that separate two homogeneous magnetic domains aligned antiparallel to each other. They fall in two large classes. In one-dimensional systems (i.e., the width of the magnetic wire is not much larger than the magnetic exchange length) DWs can be treated as one-dimensional objects called transverse walls (TW) and are classified into Néel walls $(\mathrm{NW}$ - when the magnetization rotates within the plane of the DW) and Bloch walls (BW-when the magnetization turns out of the DW plane). The influence of the current on the DW dynamics has been widely studied during the past decade for different geometries [5,10,39]. In quasi-one-dimensional stripes (i.e., the size of the system is larger than the magnetic exchange length), magnetic vortex walls (VW) appear. It has been shown that under some threshold current the VW speed scales linearly with the flowing charge current [40]. Above this threshold the profile of the magnetization is no longer rigid and there is a nucleation of more complex DWs such as vortex or antivortex walls. An important aspect in the study of the interaction of current and magnetization is the limited impact of the Oersted field produced by the current in long magnetic stripes [23,41-43], compared to wider geometries such as magnetic disks [44-47].

Another interesting class of magnetic solitons with potential application in data storage [20] concerns the magnetic skyrmions that have a large range of characteristic sizes depending on material and geometry [15-19,48-50] and can be potentially influenced by the diffusive character of the spin density [30]. The way we choose to study the diffusive effects is to introduce directly into LLG the torques coming from Zhang and $\mathrm{Li}$ [26] augmented by a spin dephasing term [8,51-53]. This treatment is general because it relies on the spin-continuity equation, independent of the details of the magnetization dynamics [54].

This paper is organized as follows. In the second section we present the spin diffusion model and we find the spin diffusion torques produced by the electrons on magnetization. In the third section we study the steady velocity effects using the Thiele formalism in the approximation where the textures are neither deformed nor rotated by the torque. We calculate also the coefficients appearing in the Thiele equation for domain walls and skyrmions. In the fourth section we conclude with the discussion and perspectives.

\section{SPIN RELAXATION MODEL AND SPIN DIFFUSION TORQUES}

The magnetization vector at the saturated magnetization $M_{s}$ can be written as $\boldsymbol{M}(\boldsymbol{x}, t)=M_{s} \boldsymbol{m}(\boldsymbol{x}, t)$, where $\boldsymbol{m}(\boldsymbol{x}, t)$ is a unitary vector varying in space $\boldsymbol{x}$ and time $t$ and $M_{s}$ is the saturation magnetization. The magnetization turns out to be proportional to the adimensional classical spin $\boldsymbol{S}(\boldsymbol{x}, t)=$ $\langle\hat{\boldsymbol{S}}(\boldsymbol{x}, t)\rangle$ as $\boldsymbol{m}=-\boldsymbol{S} / S, \hat{\boldsymbol{S}}$ being the quantum operator describing the localized spin with $S$ the corresponding molecular spin magnitude. The interaction between the localized spin $S$ and the itinerant one is of ferromagnetic nature and gives rise to the effective interaction term:

$$
\hat{H}_{\mathrm{sd}}=\Delta_{\mathrm{ex}} \boldsymbol{m} \cdot \hat{\boldsymbol{\sigma}},
$$

where $\hat{\sigma}$ is the vector of Pauli spin matrices representing spin angular momentum of the itinerant electrons, and $\Delta_{\mathrm{ex}}$ is the exchange energy between local and itinerant spins. The spin density of the itinerant electrons is defined from $\hat{\boldsymbol{\sigma}}$ as $\boldsymbol{s}=$ $\mu_{B} \operatorname{Tr}\{\hat{\rho}(\boldsymbol{m}) \hat{\boldsymbol{\sigma}}\}\left(\mu_{B}\right.$ the Bohr magneton) with $\hat{\rho}(\boldsymbol{m})$ the itinerant electron density matrix that depends on the local magnetization $\boldsymbol{m}(\boldsymbol{r}, t)$. The spin dynamics of conduction electrons can be written as a continuity equation [54] using the spin current tensor $\mathcal{J}$, which is the expectation value of the spin current tensor $\mathcal{J}=\mu_{B} \operatorname{Tr}\{\hat{\rho}(\boldsymbol{m}) \hat{\boldsymbol{v}} \otimes \hat{\boldsymbol{\sigma}}\}[26]$

$$
\frac{\partial \boldsymbol{s}}{\partial t}+\nabla \cdot \mathcal{J}=-\frac{1}{\tau_{\mathrm{ex}}} \delta \boldsymbol{s} \times \boldsymbol{m}-\frac{\delta \boldsymbol{s}}{\tau_{\mathrm{sf}}}-\frac{1}{\tau_{\phi}} \boldsymbol{m} \times(\delta \boldsymbol{s} \times \boldsymbol{m}),
$$

where $\delta \boldsymbol{s}$ is the nonequilibrium part of the spin density (see below), $\tau_{\mathrm{ex}}=\hbar S /\left(2 \Delta_{\mathrm{ex}}\right)$ is the spin precession time ( $\approx 1-10 \mathrm{fs}$ for typical ferromagnets), and $\tau_{\mathrm{sf}}$ is the spin-flip relaxation time $(\approx 0.1-1 \mathrm{ps})$ which takes into account the relaxation of the spin density $s[26]$. We have added also a term recently proposed in Ref. [53] and that takes into account the spin dephasing term proportional to $1 / \tau_{\phi}$. The torque produced by this term can be seen (due to the unitarity of $\boldsymbol{m}$ ) as a renormalization of the effect of the spin flip constant. The spin density is explicitly written as

$$
\boldsymbol{s}=n_{s} \boldsymbol{m}+\delta \boldsymbol{s},
$$

$n_{s}$ being the equilibrium spin density $\left(n_{s} \approx 10^{-2} M_{s}\right)$.

The spin current can be parsed in two contributions, $\mathcal{J}=$ $\mathcal{J}_{0}+\delta \mathcal{J}$. The spin current has a lowest order contribution which is proportional to the effective velocity of the electrons $\boldsymbol{v}_{s}$ as $\mathcal{J}_{0}=\boldsymbol{v}_{s} \otimes \boldsymbol{M}$. Explicitly, we can write $\boldsymbol{v}_{s}=-b_{J} \boldsymbol{u}_{s}$ where $b_{J}=\mu_{B} P G_{0} E / M_{s} e$, with $P$ being the spin polarization, $G_{0}$ the conductivity, $E$ the strength of the external electrical field, and $\boldsymbol{u}_{s}$ the direction of the injected current. In conventional transition metal ferromagnets, the velocity $b_{J}$ is around $10^{2} \mathrm{~m} / \mathrm{s}[26,30]$. This lowest order contribution to the spin current is called the adiabatic contribution and is responsible for the spin-transfer torque and the $\beta$ term [26]. The next contribution $\delta \mathcal{J}$ coming from the variations of the nonequilibrium part of the spin density is written as 
$\delta \mathcal{J}=-D_{0} \nabla \delta \boldsymbol{s}$, where $D_{0}$ is the spin diffusion constant which is around $\sim 10^{-2} \mathrm{~m}^{2} / \mathrm{s}[26,30,35]$.

Considering a uniform electrical current (that is to say without spatial variation) and exploiting the definition of the spin current given above, the divergence of the spin current density reduces to:

$$
\nabla \cdot \mathcal{J}=\nabla \cdot\left(\boldsymbol{v}_{s} \otimes \boldsymbol{M}-D_{0} \nabla \delta \boldsymbol{s}\right)=\left(\boldsymbol{v}_{s} \cdot \nabla\right) \boldsymbol{M}-D_{0} \nabla^{2} \delta \boldsymbol{s} .
$$

By injection of the right-hand sides of Eqs. (3) and (4) into Eq. (2), we can find an equation relating $\delta \boldsymbol{s}$ and $\boldsymbol{m}$.

In order to simplify a little the notation we define the differential operator $\mathcal{D}$ (in units of magnetization over time) as

$$
\mathcal{D}=\left(n_{s} \partial_{t}+M_{s} \boldsymbol{v}_{s} \cdot \nabla\right) .
$$

With all these considerations, and neglecting the term proportional to the temporal derivative in the nonequilibrium spin density $\partial_{t} \delta s$, it is obtained [26] that the nonequilibrium part of the spin density $\delta s$ fulfills the vector field equation

$$
D_{0} \nabla^{2} \delta \boldsymbol{s}-\frac{1}{\tau_{\mathrm{ex}}} \delta \boldsymbol{s} \times \boldsymbol{m}-\frac{\delta \boldsymbol{s}}{\tau_{\mathrm{sf}}}-\frac{1}{\tau_{\phi}} \boldsymbol{m} \times(\delta \boldsymbol{s} \times \boldsymbol{m})=\mathcal{D} \boldsymbol{m} .
$$

Using the fact that $\boldsymbol{m}$ is orthogonal to $\delta s$, Eq. (6) reduces to:

$$
\delta \boldsymbol{s}=\tau_{\mathrm{ex}} \mathcal{D} \boldsymbol{m} \times \boldsymbol{m}+\xi \delta \boldsymbol{s} \times \boldsymbol{m}-\lambda_{\mathrm{ex}}^{2} \nabla^{2} \delta \boldsymbol{s} \times \boldsymbol{m},
$$

where we have defined $\xi=\tau_{\mathrm{ex}}\left(\frac{1}{\tau_{\mathrm{sf}}}+\frac{1}{\tau_{\phi}}\right)$ and $\lambda_{\mathrm{ex}}=\sqrt{D_{0} \tau_{\mathrm{ex}}}$ is the spin precession length. Our goal is to find $\delta \boldsymbol{s}$ from this equation without completely neglecting the diffusion term that is related with the more commonly measured spin-flip diffusion length $\lambda_{\text {sf }}$ by $\lambda_{\text {ex }}=\lambda_{\mathrm{sf}} \sqrt{\tau_{\mathrm{ex}} / \tau_{\mathrm{sf}}}$, meaning that $\lambda_{\mathrm{ex}}$ is generally approximatively 10 times smaller than the spin-flip diffusion length [35]. In particular, we are interested in the diffusion effect in magnetic textures whose spin precession length is smaller (but not too much smaller) than the characteristic length $\Delta$ of the magnetic texture. For instance in $\mathrm{NiFe}$ $\lambda_{\text {ex }} \approx 1 \mathrm{~nm}$ and $\Delta$ has values ranging from 5-20 nm [55] to a few hundreds of nanometers [38]. We consider the case where $\lambda_{\text {ex }}^{2} \nabla^{2} \ll 1$, that is to say, for a magnetic texture of order $\Delta$ this limit is equivalent to $\lambda_{\text {ex }}^{2} \ll \Delta^{2}$. We perform a recursion of Eq. (7) in order to isolate the nonequilibrium spin density $\delta s$ until the linear order in $\xi \lambda_{\text {ex }}^{2} \nabla^{2}$. We find:

$$
\begin{aligned}
\delta \boldsymbol{s}= & \mathcal{C} \boldsymbol{m}+\lambda_{\mathrm{ex}}^{2} \tilde{\tau}_{\mathrm{ex}}\left[-\nabla^{2}(\mathcal{D} \boldsymbol{m} \times \boldsymbol{m}) \times \boldsymbol{m}\right. \\
& \left.+\tilde{\xi} \nabla^{2}(\mathcal{D} \boldsymbol{m} \times \boldsymbol{m})+\tilde{\xi}\left(\nabla^{2} \mathcal{D} \boldsymbol{m}\right) \times \boldsymbol{m}\right],
\end{aligned}
$$

where we have defined

$$
\mathcal{C} \boldsymbol{m}=\tilde{\tau}_{\mathrm{ex}}(\mathcal{D} \boldsymbol{m} \times \boldsymbol{m}-\xi \mathcal{D} \boldsymbol{m}),
$$

and where the tildes represent a multiplication by the factor $1 /\left(1+\xi^{2}\right)$. Under the micromagnetic assumption, the time and spatial derivatives of the magnetization $\boldsymbol{m}$ lie in a plane perpendicular to $\boldsymbol{m}$, such that $\boldsymbol{m} \cdot \mathcal{D} \boldsymbol{m}=0$ [56]. The first term of the second line of Eq. (8) can actually have contributions not orthogonal to $\boldsymbol{m}$ producing a renormalization of $n_{s}$ but not exerting torque over the magnetization. The torque exerted by the flowing spin-polarized electrons is

$$
\boldsymbol{T}=\left(1 / \tau_{\mathrm{ex}}\right) \delta \boldsymbol{s} \times \boldsymbol{m}+\left(1 / \tau_{\phi}\right) \boldsymbol{m} \times(\delta \boldsymbol{s} \times \boldsymbol{m}),
$$

and after some algebra reads $\boldsymbol{T}=\boldsymbol{T}_{0}+\boldsymbol{T}_{D}$, where we have defined

$$
\boldsymbol{T}_{0}=\frac{-1-\xi \chi}{1+\xi^{2}} \mathcal{D} \boldsymbol{m}+\frac{\chi-\xi}{1+\xi^{2}} \mathcal{D} \boldsymbol{m} \times \boldsymbol{m},
$$

where $\chi=\tau_{\mathrm{ex}} / \tau_{\phi}$. This torque reduces to the one derived by Zhang and Li [26] in the limit $\tau_{\phi} \rightarrow \infty$. The diffusive nonadiabatic torque has four contributions $\boldsymbol{T}_{D}=\sum_{i} \boldsymbol{T}_{i}$. Explicitly, these four new terms are (the first and the third were found in Ref. [30])

$$
\begin{gathered}
\boldsymbol{T}_{1}=\tilde{\lambda}_{\mathrm{ex}}^{2}(1+\tilde{\xi} \chi) \nabla^{2}(\mathcal{D} \boldsymbol{m} \times \boldsymbol{m}), \\
\boldsymbol{T}_{2}=\tilde{\lambda}_{\mathrm{ex}}^{2}(\tilde{\xi}-\chi) \nabla^{2}(\mathcal{D} \boldsymbol{m} \times \boldsymbol{m}) \times \boldsymbol{m}, \\
\boldsymbol{T}_{3}=-\tilde{\lambda}_{\mathrm{ex}}^{2} \tilde{\xi} \nabla^{2}(\mathcal{D} \boldsymbol{m}),
\end{gathered}
$$

and

$$
\boldsymbol{T}_{4}=\tilde{\lambda}_{\mathrm{ex}}^{2} \tilde{\xi} \chi \nabla^{2}(\mathcal{D} \boldsymbol{m}) \times \boldsymbol{m} .
$$

The temporal dependence of the standard torques renormalizes the gyromagnetic ratio as well as the Gilbert damping constant [27]. In transition metals this renormalization can be neglected and so the temporal dependence, such that $\mathcal{D} \boldsymbol{m}=M_{s}\left(\boldsymbol{v}_{s} \cdot \nabla\right) \boldsymbol{m}$. In this way the torques become

$$
\boldsymbol{T}_{0}=-M_{s}\left[\alpha_{0}\left(\boldsymbol{v}_{s} \cdot \nabla\right) \boldsymbol{m}+\beta\left(\boldsymbol{v}_{s} \cdot \nabla\right) \boldsymbol{m} \times \boldsymbol{m}\right],
$$

with $\alpha_{0}=(1+\xi \chi) /\left(1+\xi^{2}\right), \beta=(\xi-\chi) /\left(1+\xi^{2}\right)$. The diffusive torques become

$$
\begin{gathered}
\boldsymbol{T}_{1}=M_{s} \alpha_{1} \nabla^{2}\left[\left(\boldsymbol{v}_{s} \cdot \nabla\right) \boldsymbol{m} \times \boldsymbol{m}\right], \\
\boldsymbol{T}_{2}=M_{s} \alpha_{2} \boldsymbol{m} \times \nabla^{2}\left[\left(\boldsymbol{v}_{s} \cdot \nabla\right) \boldsymbol{m} \times \boldsymbol{m}\right], \\
\boldsymbol{T}_{3}=-M_{s} \alpha_{3} \nabla^{2}\left[\left(\boldsymbol{v}_{s} \cdot \nabla\right) \boldsymbol{m}\right], \\
\boldsymbol{T}_{4}=M_{s} \alpha_{4} \boldsymbol{m} \times \nabla^{2}\left[\left(\boldsymbol{v}_{s} \cdot \nabla\right) \boldsymbol{m}\right],
\end{gathered}
$$

with the definitions $\alpha_{1}=\tilde{\lambda}_{\text {ex }}^{2}(1+\tilde{\xi} \chi), \alpha_{2}=\tilde{\lambda}_{\text {ex }}^{2}(\chi-\tilde{\xi}), \alpha_{3}=$ $\tilde{\lambda}_{\text {ex }}^{2} \tilde{\xi}$, and $\alpha_{4}=\tilde{\lambda}_{\text {ex }}^{2} \chi \tilde{\xi}$. Until now the diffusive torques of Eqs. (13)-(16) are completely general and constitute the most important result of the present work. It is interesting to note that in spite of their similar functional form they differ in the parameters, showing that $\boldsymbol{T}_{1}$ is a pure diffusive torque (subsisting even in the case $\xi, \chi=0$ ) and $\boldsymbol{T}_{4}$ is a pure second order torque in the nonadiabatic parameters, which can be neglected when spin dephasing is small. In order to study the first-order effects of the torques, we explore their impact in the Thiele equation.

\section{DYNAMICAL EFFECTS: THIELE EQUATION FOR STEADY MOTION OF TWO-DIMENSIONAL TEXTURES}

We use the Thiele formalism for the collective coordinates of magnetization textures under the assumption of rigid movement, that is to say, in the approximation where the textures move without deformation. To study the dynamics of the magnetization one introduces the torques Eqs. (12)-(16), 
into the LLG equation

$$
\frac{\partial \boldsymbol{M}}{\partial t}=\gamma \boldsymbol{H}_{\mathrm{eff}} \times \boldsymbol{M}+\frac{\alpha}{M_{s}} \boldsymbol{M} \times \frac{\partial \boldsymbol{M}}{\partial t}+\boldsymbol{T}_{0}+\boldsymbol{T}_{D},
$$

where $\gamma$ is the gyromagnetic ratio, $\alpha$ is the Gilbert damping, and $\boldsymbol{H}_{\text {eff }}$ is the effective magnetic field coming from the functional derivative of the Hamiltonian $\mathcal{H}[\boldsymbol{M}(\boldsymbol{x})]$ as $\boldsymbol{H}_{\text {eff }}=$ $-\delta \mathcal{H}[\boldsymbol{M}(\boldsymbol{x})] / \delta \boldsymbol{M}(\boldsymbol{x})$ and it contains all the information about the properties of the material and system geometry (such as anisotropies, dipolar energy, exchange energy, etc). Using the steady state motion ansatz $\boldsymbol{M}(\boldsymbol{r}, t)=\boldsymbol{M}\left(\boldsymbol{r}-\boldsymbol{v}_{d} t\right)$, Eq. (17) and Eqs. (13)-(16) LLG can be rewritten as:

$$
\begin{aligned}
\gamma \boldsymbol{H}_{\mathrm{eff}, \perp}= & \boldsymbol{m} \times\left(\left(\alpha_{0} \boldsymbol{v}_{s}-\boldsymbol{v}_{d}\right) \cdot \nabla\right) \boldsymbol{m}+\left(\left(\beta \boldsymbol{v}_{s}-\alpha \boldsymbol{v}_{d}\right) \cdot \nabla\right) \boldsymbol{m} \\
& +\alpha_{1} \nabla^{2}\left[\left(\boldsymbol{v}_{s} \cdot \nabla\right) \boldsymbol{m} \times \boldsymbol{m}\right] \times \boldsymbol{m} \\
& +\alpha_{2} \nabla^{2}\left[\left(\boldsymbol{v}_{s} \cdot \nabla\right) \boldsymbol{m} \times \boldsymbol{m}\right] \\
& -\alpha_{3} \nabla^{2}\left[\left(\boldsymbol{v}_{s} \cdot \nabla\right) \boldsymbol{m}\right] \times \boldsymbol{m}+\alpha_{4} \nabla^{2}\left[\left(\boldsymbol{v}_{s} \cdot \nabla\right) \boldsymbol{m}\right] .
\end{aligned}
$$

To obtain the Thiele equation [57] we have to project Eq. (18) into the generator of the translational mode $\left(\partial_{\beta} m_{\alpha}\right)$ [58], so we obtain four diffusive terms linear in the electron velocity as $\tau_{i, \alpha}\left(\partial_{\beta} m_{\alpha}\right)=v_{s l} T_{l \beta}^{i}$ (with $\left.i=1,2,3,4\right)$ that are added to the spin transfer and the $\beta$ term. After integration in the whole two-dimensional domain the equation becomes

$$
\boldsymbol{G}\left(\alpha_{0} \boldsymbol{v}_{s}-\boldsymbol{v}_{d}\right)+\boldsymbol{D}\left(\beta \boldsymbol{v}_{s}-\alpha \boldsymbol{v}_{d}\right)+\mathcal{T} \boldsymbol{v}_{s}=\boldsymbol{F}
$$

with the tensors $G_{l \beta}=\int d^{2} r \boldsymbol{m} \cdot\left(\partial_{l} \boldsymbol{m} \times \partial_{\beta} \boldsymbol{m}\right), \quad D_{l \beta}=$ $\int d^{2} r\left(\partial_{l} \boldsymbol{m} \cdot \partial_{\beta} \boldsymbol{m}\right)$, and $\mathcal{T}=\alpha_{1} \mathcal{T}^{1}+\alpha_{2} \mathcal{T}^{2}+\alpha_{3} \mathcal{T}^{3}+\alpha_{4} \mathcal{T}^{4}$, where

$$
\begin{aligned}
& \mathcal{T}_{l \beta}^{1}=-\int d^{2} r \nabla^{2}\left(\partial_{l} \boldsymbol{m} \times \boldsymbol{m}\right) \cdot\left(\partial_{\beta} \boldsymbol{m} \times \boldsymbol{m}\right), \\
& \mathcal{T}_{l \beta}^{2}=\int d^{2} r \nabla^{2}\left(\partial_{l} \boldsymbol{m} \times \boldsymbol{m}\right) \cdot \partial_{\beta} \boldsymbol{m}, \\
& \mathcal{T}_{l \beta}^{3}=\int d^{2} r\left(\nabla^{2}\left(\partial_{l} \boldsymbol{m}\right) \times \partial_{\beta} \boldsymbol{m}\right) \cdot \boldsymbol{m}, \\
& \mathcal{T}_{l \beta}^{4}=\int d^{2} r \nabla^{2}\left(\partial_{l} \boldsymbol{m}\right) \cdot \partial_{\beta} \boldsymbol{m} .
\end{aligned}
$$

The vector $\boldsymbol{F}$ is a pinning force due to the (possible) impurities or boundary effects. In a translational invariant system $\boldsymbol{F}$ vanishes and all the contributions acting as forces are the terms proportional to $\boldsymbol{v}_{s}$. The tensor $\boldsymbol{G}$ is known as the gyrotropic tensor. It is different from zero and antisymmetric in the case of two-dimensional textures with dependence in $x$ and $y$ such as VWs, vortex cores, and skyrmions, and it vanishes in walls having a trivial dependence in one of the spatial directions, such as TWs. In two spatial dimensions the tensors $\mathcal{T}^{i}$ are also $2 \times 2$. The Thiele equation can be solved explicitly for $\boldsymbol{F}=0$ as $\boldsymbol{v}_{d}=\boldsymbol{V} \boldsymbol{v}_{s}$ where the tensor $\boldsymbol{V}$ is

$$
\boldsymbol{V}=(\boldsymbol{G}+\alpha \boldsymbol{D})^{-1}\left(\alpha_{0} \boldsymbol{G}+\beta \boldsymbol{D}+\mathcal{T}\right) .
$$

In what follows we will study how spin diffusion modifies the spin torque for different textures trying to keep the analysis as general as possible.

\section{A. Transverse walls}

TWs are magnetic textures where the magnetization at the interface between two asymptotic regions has homogeneous remains in a defined plane (characterized by a constant azimuthal angle). In principle, in the flow regime the steadystate DW velocity $v$ for any DW structure reads $v_{d, \|}=(\beta / \alpha) v_{s}$ [23], and then the efforts have been centered in measuring this ratio. In general, the $\beta$ term contributes to nonadiabicity and depends on the magnetic texture. Claudio-González et al. [29] studied the origin of the enhanced of nonadiabacity term considering spin diffusion effects. Recently, Akosa et al. [30] studied the spin diffusion in current-induced DWs and they found two new spatially dependent torques which have to be added to LLG equation. This helps to understand the role of the spin diffusion in the dynamics of magnetic textures. If we take a generic TW varying along the $x$ axis the simplest solution of Eq. (19) is found simply by realizing that the diffusive torque tensors have nonzero components only in $x x$. Hence, applying a current in the $x$ axis the solution for the wall velocity is simply

$$
\boldsymbol{v}_{d}=\left(\frac{\beta}{\alpha}+\kappa\right) v_{s} \hat{\boldsymbol{x}}
$$

where $\kappa \equiv\left(\alpha \mathcal{T}_{x x} / D_{x x}\right)$ corrects the steady velocity found in the parameter region where the wall is not deformed or rotated, that is to say, before the Walker breakdown where $\Phi(t)$ acquires temporal dependence [39].

In order to calculate explicitly the parameter $\kappa$ we consider the simplest TW, that is to say, the NW [40,59]. If the variation is along one axis we can choose the plane of the magnetization as $\Theta(\boldsymbol{x})=\pi / 2$ and the NW wall along $x$ can be expressed as the Walker trial function [40]:

$$
\Phi(x)=2 \arctan \left[\exp \left(\frac{x}{\Delta}\right)\right] .
$$

Using Eq. (23) we can show that the only torques contributing to the correction are $\mathcal{T}_{x x}^{1}$ and $\mathcal{T}_{x x}^{4}$. In other words, the correction is of purely diffusive origin at the first order (the terms proportional to nonadiabaticity are quadratic as $\sim \xi \chi)$. For TWs, we find explicitly (neglecting second order terms):

$$
\kappa=\frac{\alpha}{3}\left(\frac{\lambda_{\mathrm{ex}}}{\Delta}\right)^{2}
$$

so we can see that for NWs the effects are inversely proportional to the square of the wall width and more important for materials whose walls are smaller than the spin precession length. The spin diffusion clearly enhances the effective nonadiabaticity of the system, in complete agreement with Refs. [29,30]. The enhancement can actually be very significant for small domain wall and large spin precession lengths (i.e., weak ferromagnets). The same calculation can be done in the BW case, by setting $\Theta(x)=2 \arctan \left[\exp \left(\frac{x}{\Delta}\right)\right]$ and $\Phi(\boldsymbol{x})=\pi / 2$ founding exactly the same result for the evaluated torques and therefore the same value Eq. (24) for the correcting term $\kappa$. These results complete previous works where the nonadiabaticity ( $\beta$ constant) was predicted to depend on spin accumulation and to have an influence in the velocity of the domain walls. Numerical simulations can be performed 
to test the effects of these new torques for more complex DWs as vortex walls.

\section{B. Axisymmetric cases}

We can have an intuitive idea of the role of the new torques considering a texture $\boldsymbol{m}(\boldsymbol{x})=(\cos (\Phi(\boldsymbol{x}))$ $\sin (\Theta(\boldsymbol{x})), \sin (\Phi(\boldsymbol{x})) \sin (\Theta(\boldsymbol{x})), \cos (\Theta(\boldsymbol{x})))$ with axisymmetric symmetry. In other words, we consider a two-dimensional texture with rotational symmetry, which can be parameterized as $\Theta(\boldsymbol{x})=\Theta(r)$ and $\Phi(\boldsymbol{x})=p \phi+\gamma$ where $(r, \phi)$ are the plane polar coordinates, $p$ has to be an integer number and $\gamma$ is a phase that tilts the magnetization vector from the radial direction. With this ansatz the torque densities $\boldsymbol{\tau}_{i}$, defined as $\mathcal{T}^{i}=\int \boldsymbol{\tau}^{i} d^{2} x$ and appearing in Eq. (20) can be calculated in a quite general way. Using the two-dimensional matrix rotation $R_{\phi}=((\cos \phi,-\sin \phi),(\sin \phi, \cos \phi))$ it is possible to convert the torques as $\tau^{i}=R_{\phi} t^{i} R_{\phi}^{-1}$, where the matrices $t^{i}$ are diagonal or antidiagonal matrices. The explicit expression for the torques appearing in the Thiele equation after this transformation are complicated expressions but clearly show that the $t^{1}$ and $t^{4}$ matrices are diagonal while $t^{2}$ and $t^{3}$ are antidiagonal. This can be directly seen from the parity of the torques with respect to magnetization reversal: $t^{1}$ and $t^{4}$ are even in magnetization and therefore enhance the nonadiabaticity, while $t^{2}$ and $\boldsymbol{t}^{3}$ are odd and renormalize the adiabatic torque. In this way we can expect a dissipative effect coming from $\boldsymbol{\tau}^{1}$ and $\boldsymbol{\tau}^{4}$ (similar to the effect produced by the $\boldsymbol{D}$ tensor) and a gyrotropic effect coming from $\boldsymbol{\tau}^{2}$ and $\boldsymbol{\tau}^{3}$ as we show in the next subsection for the particular case of skyrmions.

\section{Skyrmion}

Let us consider the particular case of a Belavin-Polyakov (BP) skyrmion [60,61] in which $\Theta_{\mathrm{BP}}(r)=2 \arctan \left(\left(\frac{r}{r_{0}}\right)^{q}\right)$, and as before $\Phi(\boldsymbol{x})=p \phi+\gamma$ where $r_{0}$ is the size of the core of the skyrmion, $q$ is an integer. The skyrmionic topological charge is $Q=p q$. We can explicitly calculate the functions for the torque densities in the Thiele equation. The simplest case $q=p=1$ gives us the same function $f(r)$ for all the elements of the torque densities

$$
f(r)=\frac{1}{r_{0}^{4}} \frac{32}{\left(1+\left(\frac{r}{r_{0}}\right)^{2}\right)^{4}},
$$

and $\boldsymbol{\tau}^{1}=-\boldsymbol{\tau}^{4}=f(r) \mathbb{I}$, and $\boldsymbol{\tau}^{2}=\boldsymbol{\tau}^{3}=f(r) R_{\pi / 2}$, where $\mathbb{I}$ is the $2 \times 2$ unitary matrix and $R_{\pi / 2}$ is the $2 \times 2$ antisymmetric matrix. Performing the two-dimensional integration $\int f(r) r d r d \phi=32 \pi /\left(3 r_{0}^{2}\right)$ we found readily the expression for the torques in the BP skyrmion case

$$
\mathcal{T}=\frac{32 \pi}{3}\left(\frac{\tilde{\lambda}_{\mathrm{ex}}}{r_{0}}\right)^{2}\left(\begin{array}{cc}
1 & -\beta^{\prime} \\
\beta^{\prime} & 1
\end{array}\right),
$$

where we have defined $\beta^{\prime}=(2 \tilde{\xi}-\chi)$. We can see from this result that the spin diffusion term $\mathcal{T}$ has a twofold effect in the skyrmion dynamics: first an enhancement (diagonal term proportional to the spin precession length) of the dispersive

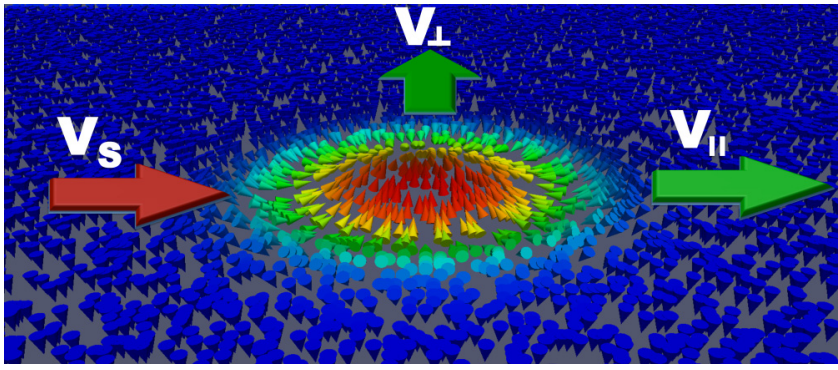

FIG. 1. Schematic of the movement of the skyrmion when submitted to an electrical current. As is depicted in the figure, electrons injected with velocity $\boldsymbol{v}_{s}$ produce a longitudinal $\boldsymbol{v}_{\|}$and a perpendicular $\boldsymbol{v}_{\perp}$ movement of the soliton.

effect of the $\boldsymbol{D}$ tensor

$$
\boldsymbol{D}=4 \pi\left(\begin{array}{ll}
1 & 0 \\
0 & 1
\end{array}\right),
$$

plus a reduction (antidiagonal term) of the gyrotropic effect coming from the $\boldsymbol{G}$ tensor:

$$
\boldsymbol{G}=4 \pi\left(\begin{array}{cc}
0 & 1 \\
-1 & 0
\end{array}\right)
$$

With these results we can calculate explicitly the matrix $\boldsymbol{V}=$ $\boldsymbol{V}_{0}+\boldsymbol{V}_{\lambda_{\text {ex }}}$ of Eq. (21) with

$$
\begin{gathered}
\boldsymbol{V}_{0}=\frac{1}{\left(1+\alpha^{2}\right)}\left(\begin{array}{cc}
\alpha_{0}+\alpha \beta & \alpha_{0} \alpha-\beta \\
\beta-\alpha_{0} \alpha & \alpha_{0}+\alpha \beta
\end{array}\right), \\
\boldsymbol{V}_{\lambda_{\mathrm{ex}}}=\frac{8}{3\left(1+\alpha^{2}\right)}\left(\frac{\tilde{\lambda}_{\mathrm{ex}}}{r_{0}}\right)^{2}\left(\begin{array}{cc}
\alpha-\beta^{\prime} & -\left(1+\alpha \beta^{\prime}\right) \\
1+\alpha \beta^{\prime} & \alpha-\beta^{\prime}
\end{array}\right) .
\end{gathered}
$$

In the absence of spin diffusion $(\mathcal{T}=0)$ we can see from Eq. (29) that $\alpha_{0}=1$ (that is to say when the term $\chi \xi$ is neglected) and $\alpha=\beta$ implies $\boldsymbol{v}_{d}=\boldsymbol{v}_{s}$ (as it is verified also in the case of TWs) which means that in that case the texture moves in the same direction and with the same magnitude as the effective electron speed. When considering the spin diffusion we can observe that the role of the difference $\alpha-\beta^{\prime}$ is different in the spin diffusion matrix [Eq. (30)] compared to the nondiffusive matrix [Eq. (29)]. As a consequence even in the so-called Galilean limit $\alpha=\beta^{\prime}$ we have a contribution to the Magnus effect and a perpendicular contribution to the velocity of the skyrmion (Fig. 1). This is easily illustrated choosing a velocity in the $x$ direction and writing the velocity of the structure using Eqs. (29) and (30), finding

$$
\begin{aligned}
& \frac{v_{\|}}{\left|v_{s}\right|}=\frac{1}{1+\alpha^{2}}\left[\alpha_{0}+\alpha \beta+\frac{8}{3}\left(\frac{\tilde{\lambda}_{\mathrm{ex}}}{r_{0}}\right)^{2}\left(\alpha-\beta^{\prime}\right)\right], \\
& \frac{v_{\perp}}{\left|v_{s}\right|}=\frac{1}{1+\alpha^{2}}\left[\beta-\alpha_{0} \alpha+\frac{8}{3}\left(\frac{\tilde{\lambda}_{\mathrm{ex}}}{r_{0}}\right)^{2}\left(1+\alpha \beta^{\prime}\right)\right] .
\end{aligned}
$$

It is interesting to note that while the correction in the parallel velocity is proportional to the difference $\left(\alpha-\beta^{\prime}\right)$ (and then it is not necessarily positive), the correction in the perpendicular term is proportional to $\left(1+\alpha \beta^{\prime}\right)$ that always contributes to increasing the Magnus effect, at least in the generical case $\beta^{\prime}>0(2 \tilde{\xi}>\chi)$. In order to have a more precise idea of the behavior of the transversal and longitudinal velocity we use the approximative skyrmion parameters found 
(a)

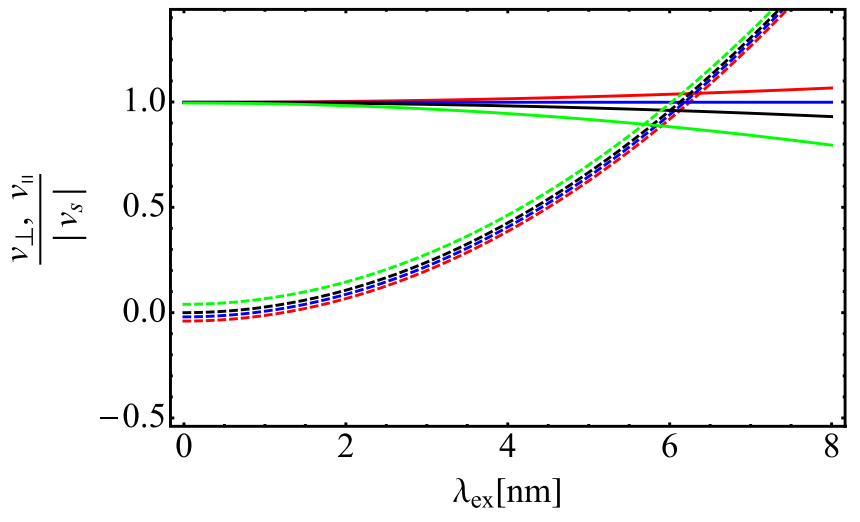

(b)

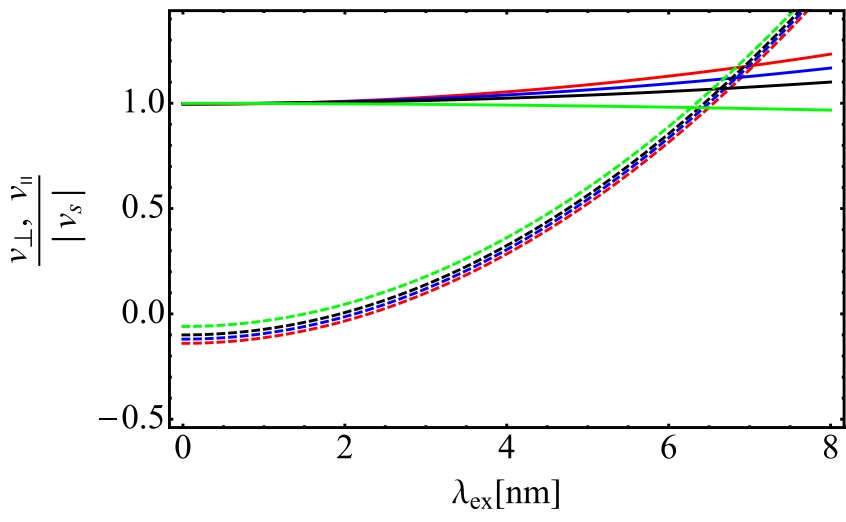

FIG. 2. Dependence of the longitudinal (solid lines) and transverse (dotted lines) velocity of a Belavin-Polyakov skyrmion, as a function of the spin precession length $\lambda_{\text {ex }}$ for (a) $\chi=0$ and (b) $\chi=0.1$. The parameters $\alpha=0.04, r_{0}=10 \mathrm{~nm}$, and different values of $\xi=0,0.5 \alpha, \alpha, 2 \alpha$ (red, blue black, green, respectively). The velocity is given in units of the effective electron velocity $\left|v_{s}\right|$.

in Iwasaki et al. [21] for skyrmions in $\mathrm{MnSi}$ and depicted the dependence on $\lambda_{\text {ex }}$ in Fig. 2 for two different values of $\chi$. We found a significant enhancement of the perpendicular velocity upon increasing the spin precession length $\lambda_{\mathrm{ex}}$ (or, equivalently, upon reducing the skyrmion size) and so a larger deflection of the skyrmion center, as we see in Fig. 2. In other words, the Hall angle defined as $R=v_{\perp} / v_{\|}[21,62]$ is larger for larger values of $\beta^{\prime}$ and $\lambda_{\text {ex }}$.
Similar effects are expected for magnetic vortices, the main difficulty being the absence of analytical solutions without singularities in the derivatives, as the Usov solution [63] or other piecewise solutions [64]. This difficulty can be overcome by numerical simulations, but such an approach exceeds the scope of the present work.

\section{CONCLUSIONS AND PERSPECTIVES}

The main contribution of the present work is to establish the exact functional form of the spin diffusion torques appearing in LLG when considering the semiclassical model for the nonequilibrium spin density appearing in the works of Zhang and $\mathrm{Li}$ [26] plus the spin dephasing effect. The torques appearing in LLG are calculated. These torques are the linear contribution of the spin diffusion, and they are in principle general up to the third order in magnetization gradient. In this sense the clear expression of these new terms allows us to explore the diffusive effect in the classical limit for any magnetic texture and in a general frame. In order to explore some effects of these terms we have deduced the appearance of the diffusive torques in the case of two-dimensional textures, in the limit of rigid motion. The simplest case of single skyrmions and transverse walls shows that the main effect of diffusion is an increase of the nonadiabaticity and so a larger longitudinal velocity for TWs and a larger Magnus effect for the case of skyrmions. These results can be tested experimentally when measuring the velocities of the skyrmion and domain walls. In effect, as the experimental values of the diffusion constant can be found tabulated in the literature [35], our results can help to determine the exact values of the nonadiabatic parameters $\beta$ or the spin dephasing constant $\chi$. Numerical analysis of LLG is also expected in following works to test these predictions and also to explore the cases where the approximative analytical expressions do not allow us to perform reliable analytical calculations (mainly because of the discontinuity in derivatives, viz as in vortex cores and VWs cases).

\section{ACKNOWLEDGMENTS}

R.G.E. thanks Conicyt Pai/Concurso Nacional de Apoyo al Retorno de Investigadores/as desde el Extranjero Folio 821320024. N.V.-S. thanks CONICYT-PCHA/Doctorado Nacional/2014-21140141. A.M. was supported by the King Abdullah University of Science and Technology (KAUST).
[1] S. S. P. Parkin, M. Hayashi, and L. Thomas, Science 320, 190 (2008).

[2] C. Chappert, A. Fert, and F. N. Van Dau, Nat. Mater. 6, 813 (2007).

[3] I. Žutić, J. Fabian, and S. Das Sarma, Rev. Mod. Phys. 76, 323 (2004).

[4] C. H. Marrows, Adv. Phys. 54, 585 (2005).

[5] O. Boulle, G. Malinowski, and M. Kläui, Mater. Sci. Eng., R 72, 159 (2011).

[6] J. C. Slonczewski, J. Magn. Magn. Mater. 159, L1 (1996).
[7] L. Berger, Phys. Rev. B 54, 9353 (1996).

[8] M. D. Stiles and A. Zangwill, Phys. Rev. B 66, 014407 (2002).

[9] L. D. Landau and E. M. Lifshitz, Z. Sowj. 8, 153 (1935).

[10] K.-J. Lee, M. Stiles, H.-W. Lee, J.-H. Moon, K.-W. Kim, and S.-W. Lee, Phys. Rep. 531, 89 (2013).

[11] C. Abert, M. Ruggeri, F. Bruckner, C. Vogler, G. Hrkac, D. Praetorius, and D. Suess, Sci. Rep. 5, 14855 (2015).

[12] M. Sturma, C. Bellegarde, J.-C. Toussaint, and D. Gusakova, Phys. Rev. B 94, 104405 (2016). 
[13] A. M. Kosevich, B. A. Ivanov, and A. S. Kovalev, Phys. Rep. 194, 117 (1990).

[14] K. Yamada, S. Kasai, Y. Nakatani, K. Kobayashi, H. Kohno, A. Thiaville, and T. Ono, Nat. Mater. 6, 270 (2007).

[15] W. Jiang, P. Upadhyaya, W. Zhang, G. Yu, M. B. Jungfleisch, F. Y. Fradin, J. E. Pearson, Y. Tserkovnyak, K. L. Wang, O. Heinonen, S. G. E. te Velthuis, and A. Hoffmann, Science 349, 283 (2015).

[16] O. Boulle, J. Vogel, H. Yang, S. Pizzini, D. de Souza Chaves, A. Locatelli, T. O. Menteş, A. Sala, L. D. Buda-Prejbeanu, O. Klein, M. Belmeguenai, Y. Roussigné, A. Stashkevich, S. M. Chérif, L. Aballe, M. Foerster, M. Chshiev, S. Auffret, I. M. Miron, and G. Gaudin, Nat. Nanotechnol. 11, 449 (2016).

[17] C. Moreau-Luchaire, C. Moutafis, N. Reyren, J. Sampaio, C. A. F. Vaz, N. Van Horne, K. Bouzehouane, K. Garcia, C. Deranlot, P. Warnicke, P. Wohlhüter, J.-M. George, M. Weigand, J. Raabe, V. Cros, and A. Fert, Nat. Nanotechnol. 11, 444 (2016).

[18] S. Woo, K. Litzius, B. Kruger, M.-Y. Im, L. Caretta, K. Richter, M. Mann, A. Krone, R. M. Reeve, M. Weigand, P. Agrawal, I. Lemesh, M.-A. Mawass, P. Fischer, M. Klaui, and G. S. D. Beach, Nat. Mater. 15, 501 (2016).

[19] G. Yu, P. Upadhyaya, X. Li, W. Li, S. K. Kim, Y. Fan, K. L. Wong, Y. Tserkovnyak, P. K. Amiri, and K. L. Wang, Nano Lett. 16, 1981 (2016).

[20] A. Fert, V. Cros, and J. Sampaio, Nat. Nanotechnol. 8, 152 (2013).

[21] J. Iwasaki, M. Mochizuki, and N. Nagaosa, Nat. Commun. 4, 1463 (2013).

[22] Y. B. Bazaliy, B. A. Jones, and S.-C. Zhang, Phys. Rev. B 57, R3213(R) (1998).

[23] A. Thiaville, Y. Nakatani, J. Miltat, and Y. Suzuki, EPL (Europhys. Lett.) 69, 990 (2005).

[24] R. A. Duine, A. S. Nunez, J. Sinova, and A. H. MacDonald, Phys. Rev. B 75, 214420 (2007).

[25] S. Zhang and Steven S.-L. Zhang, Phys. Rev. Lett. 102, 086601 (2009).

[26] S. Zhang and Z. Li, Phys. Rev. Lett. 93, 127204 (2004).

[27] G. Tatara and H. Kohno, Phys. Rev. Lett. 92, 086601 (2004).

[28] F. Piéchon and A. Thiaville, Phys. Rev. B 75, 174414 (2007).

[29] D. Claudio-Gonzalez, A. Thiaville, and J. Miltat, Phys. Rev. Lett. 108, 227208 (2012).

[30] C. A. Akosa, W.-S. Kim, A. Bisig, M. Kläui, K.-J. Lee, and A. Manchon, Phys. Rev. B 91, 094411 (2015).

[31] M. Johnson and R. H. Silsbee, Phys. Rev. B 35, 4959 (1987).

[32] P. C. van Son, H. van Kempen, and P. Wyder, Phys. Rev. Lett. 58, 2271 (1987).

[33] M. N. Baibich, J. M. Broto, A. Fert, F. Nguyen Van Dau, F. Petroff, P. Etienne, G. Creuzet, A. Friederich, and J. Chazelas, Phys. Rev. Lett. 61, 2472 (1988).

[34] P. M. Levy, Solid State Phys. Ser. 47, 367 (1994).

[35] J. Bass and W. P. Pratt, Jr., J. Phys.: Condens. Matter 19, 183201 (2007).

[36] C. Burrowes, A. P. Mihai, D. Ravelosona, J. V. Kim, C. Chappert, L. Vila, A. Marty, Y. Samson, F. Garcia-Sanchez,
L. D. Buda-Prejbeanu, I. Tudosa, E. E. Fullerton, and J. P. Attane, Nat. Phys. 6, 17 (2010).

[37] L. Heyne, J. Rhensius, D. Ilgaz, A. Bisig, U. Rüdiger, M. Kläui, L. Joly, F. Nolting, L. J. Heyderman, J. U. Thiele, and F. Kronast, Phys. Rev. Lett. 105, 187203 (2010).

[38] T. Trunk, M. Redjdal, A. Kákay, M. F. Ruane, and F. B Humphrey, J. Appl. Phys. 89, 7606 (2001).

[39] Y. Tserkovnyak, A. Brataas, and G. E. W. Bauer, J. Magn. Magn. Mater. 320, 1282 (2008).

[40] J. He, Z. Li, and S. Zhang, Phys. Rev. B 73, 184408 (2006).

[41] M. Kläui, P.-O. Jubert, R. Allenspach, A. Bischof, J. A. C. Bland, G. Faini, U. Rüdiger, C. A. F. Vaz, L. Vila, and C. Vouille, Phys. Rev. Lett. 95, 026601 (2005).

[42] G. Meier, M. Bolte, R. Eiselt, B. Krüger, D.-H. Kim, and P. Fischer, Phys. Rev. Lett. 98, 187202 (2007).

[43] D. Morecroft, I. A. Colin, F. J. Castaño, J. A. C. Bland, and C. A. Ross, Phys. Rev. B 76, 054449 (2007).

[44] G. Consolo, B. Azzerboni, G. Finocchio, L. Lopez-Diaz, and L. Torres, J. Appl. Phys. 101, 09C108 (2007).

[45] J.-H. Moon, D.-H. Kim, M. H. Jung, and K.-J. Lee, Phys. Rev. B 79, 134410 (2009).

[46] A. Dussaux, A. V. Khvalkovskiy, P. Bortolotti, J. Grollier, V. Cros, and A. Fert, Phys. Rev. B 86, 014402 (2012).

[47] J. A. Otálora, D. Cortés-Ortuño, D. Görlitz, K. Nielsch, and P. Landeros, J. Appl. Phys. 117, 173914 (2015).

[48] U. K. Roszler, A. N. Bogdanov, and C. Pfleiderer, Nature (London) 442, 797 (2006).

[49] S. Muhlbauer, B. Binz, F. Jonietz, C. Pfleiderer, A. Rosch, A. Neubauer, R. Georgii, and P. Boni, Science 323, 915 (2009).

[50] N. Nagaosa and Y. Tokura, Nat. Nanotechnol. 8, 899 (2013).

[51] M. Zwierzycki, Y. Tserkovnyak, P. J. Kelly, A. Brataas, and G. E. W. Bauer, Phys. Rev. B 71, 064420 (2005).

[52] X. Wang and A. Manchon, Phys. Rev. Lett. 108, 117201 (2012).

[53] C. Petitjean, D. Luc, and X. Waintal, Phys. Rev. Lett. 109, 117204 (2012).

[54] Q.-f. Sun and X. C. Xie, Phys. Rev. B 72, 245305 (2005).

[55] A. Thiaville and Y. Nakatani, in Spin Dynamics in Confined Magnetic Structures III, edited by B. Hillebrands and A. Thiaville, Vol. 101 (Springer, Berlin, Heidelberg, 2006), p. 161.

[56] L. D. Landau and E. M. Lifshitz, Electrodynamics of Continuous Media (Pergamon Press, Oxford, England, 1984).

[57] A. A. Thiele, Phys. Rev. Lett. 30, 230 (1973).

[58] K. Everschor, M. Garst, R. A. Duine, and A. Rosch, Phys. Rev. B 84, 064401 (2011).

[59] J. M. D. Coey, Magnetism and Magnetic Materials (Cambridge University Press, 2009).

[60] A. A. Belavin and A. M. Polyakov, JETP Lett. 22, 245 (1975).

[61] B. A. Ivanov, D. D. Sheka, V. V. Krivonos, and F. G. Mertens, Phys. Rev. B 75, 132401 (2007).

[62] T. Schulz, R. Ritz, A. Bauer, M. Halder, M. Wagner, C. Franz, C. Pfleiderer, K. Everschor, M. Garst, and A. Rosch, Nat. Phys. 8, 301 (2012).

[63] N. A. Usov and S. E. Peschany, J. Magn. Magn. Mater. 118, L290 (1993).

[64] K. L. Metlov, arXiv:cond-mat/0012146. 